\title{
Is the presence of medical trainees associated with increased mortality with weekend admission?
}

\author{
Rocco Ricciardi , Jason Nelson, Patricia L Roberts, Peter W Marcello, Thomas E Read and David J Schoetz
}

\begin{abstract}
Background: Several studies have demonstrated increased inhospital mortality following weekend admission. We hypothesized that the presence of resident trainees reduces the weekend mortality trends.

Methods: We identified all patients with a non-elective hospital admission from 1/1/2003 through 12/31/2008. We abstracted vital status on discharge and calculated the Charlson comorbidity score for all inpatients.

We compared odds of inpatient mortality following non-elective admission on a weekend day as compared to a weekday, while considering diagnosis, patient characteristics, comorbidity, hospital factors, and care at hospitals with resident trainees.

Results: Data were available for 48,253,968 patient discharges during the six-year study period. The relative risk of mortality was $15 \%$ higher following weekend admission as compared to weekday admission. After adjusting for diagnosis, age, sex, race, income level, payer, comorbidity, and weekend admission the overall odds of mortality was higher for patients in hospitals with fewer nurses and staff physicians. Mortality following a weekend admission for patients admitted to a hospital with resident trainees was significantly higher (17\%) than hospitals with no resident trainees $(p<0.001)$.

Conclusions: Low staffing levels of nurses and physicians significantly impact mortality on weekends following non-elective admission. Conversely, patients admitted to hospitals with more resident trainees had significantly higher mortality following a weekend admission.
\end{abstract}

Keywords: Mortality, Weekend, Trainee, Non-elective

\section{Background}

A growing number of studies have demonstrated increased mortality on weekends for patients suffering from several urgent medical and surgical diagnoses [1,2]. Increasingly, there is evidence suggesting that patient care, especially for those with time-sensitive conditions [3], is compromised during weekends and that patients become unnecessarily vulnerable on weekends. No clear explanation for this mortality difference exists; however a number of potential factors should be considered. Factors influencing excess weekend mortality may include reduced staff levels, wide physician cross-coverage, and

\footnotetext{
*Correspondence: rocco.ricciardi@lahey.org

Department of Colon and Rectal Surgery, Lahey Clinic, Tufts University, 41 Mall Rd, Burlington, MA 01805, USA
}

\section{Biomed Central}

(c) 2014 Ricciardi et al.; licensee BioMed Central Ltd. This is an open access article distributed under the terms of the Creative Commons Attribution License (http://creativecommons.org/licenses/by/2.0), which permits unrestricted use, distribution, and reproduction in any medium, provided the original work is properly cited. fewer specialized diagnostic, procedural, and treatment options on the weekend [3].

One of the greatest concerns regarding weekend staffing is that fewer nurses and staff physicians are available, while less-experienced caregivers provide the bulk of care. In addition, resident physicians or trainees often provide many urgent care services on nights and weekends. Given the often front line role that resident physicians have in patient care on weekends, we performed an analysis to delineate the potential effect of resident trainees on disparate outcomes based on day of admission. Our hypothesis was that resident trainees act as front line caregivers and thus, hospitals with residency programs may experience a reduced overall mortality following weekend admission. Low staffing levels and a lack of resident trainees may lead to care that is untimely 
Table 1 Demographics of patients admitted to the hospital for non-elective indications on a weekday compared with the weekends

\begin{tabular}{|c|c|c|c|}
\hline Demographics & $\begin{array}{c}\text { Weekend } \\
(\mathrm{N}=5,998,016)\end{array}$ & $\begin{array}{c}\text { Weekday } \\
(\mathrm{N}=20,053,759)\end{array}$ & $\begin{array}{c}\text { All patients } \\
(\mathrm{N}=26,051,775)\end{array}$ \\
\hline Age, mean (SD) & $47.9(29.6)$ & $46.9(29.7)$ & $47.1(29.6)$ \\
\hline \multicolumn{4}{|l|}{ Sex } \\
\hline Male & $2,651,234(23.3)$ & $8,747,217(76.7)$ & $11,398,451$ \\
\hline Female & $3,321,072(22.8)$ & $11,228,425(77.2)$ & $14,549,497$ \\
\hline Missing & $25,710(24.8)$ & $78,117(75.2)$ & 103,827 \\
\hline \multicolumn{4}{|l|}{ Race } \\
\hline White & $3,017,636(22.7)$ & $10,304,064(77.3)$ & $13,321,700$ \\
\hline Asian & 139,398 (23.6) & $452,512(76.4)$ & 591,910 \\
\hline Black & $676,910(23.3)$ & 2,232,936 (76.7) & $2,909,846$ \\
\hline Hispanic & $670,770(23.3)$ & $2,211,791(76.7)$ & $2,882,561$ \\
\hline Native American & $24,399(23.4)$ & 79,707 (76.6) & 104,106 \\
\hline Other & $142,420(23.0)$ & $478,022(77.0)$ & 620,442 \\
\hline Missing & $1,326,483(23.6)$ & $4,294,727(76.4)$ & $5,621,210$ \\
\hline \multicolumn{4}{|l|}{ Median Household Income } \\
\hline 0-25th Percentile & $1,550,655(23.1)$ & $5,171,693(76.9)$ & $6,722,348$ \\
\hline 26-50th Percentile & $1,477,013(23.1)$ & 4,919,398 (76.9) & $6,396,411$ \\
\hline 51-75th Percentile & $1,407,992(23.0)$ & $4,708,169(77.0)$ & $6,116,161$ \\
\hline 76-100th Percentile & $1,402,397(22.9)$ & $4,723,520(77.1)$ & $6,125,917$ \\
\hline Missing & $159,959(23.2)$ & $530,979(76.8)$ & 690,938 \\
\hline \multicolumn{4}{|l|}{ Primary Payer } \\
\hline Private & $1,916,698(22.3)$ & $6,693,914(77.7)$ & $8,610,612$ \\
\hline Medicaid & $1,185,764(22.8)$ & $4,018,221(77.2)$ & $5,203,985$ \\
\hline Medicare & $2,323,860(23.5)$ & $7,560,323(76.5)$ & $9,884,183$ \\
\hline None & $38,416(25.0)$ & $115,206(75.0)$ & 153,622 \\
\hline Other & $183,121(22.4)$ & $635,129(77.6)$ & 818,250 \\
\hline Self-Pay & $342,981(25.4)$ & $1,007,693(74.6)$ & $1,350,674$ \\
\hline Missing & $7,176(23.6)$ & $23,273(76.4)$ & 30,449 \\
\hline Comorbidity Score, mean (SD) & $1.1(1.7)$ & $1.1(1.7)$ & $1.1(1.7)$ \\
\hline \multicolumn{4}{|l|}{ MDC } \\
\hline Nervous System & $397,624(25.2)$ & $1,178,110(74.8)$ & $1,575,734$ \\
\hline Alcohol/Drug Use & $73,234(23.1)$ & $244,275(76.9)$ & 317,509 \\
\hline Burns & $6,909(29.0)$ & $16,888(71.0)$ & 23,797 \\
\hline Circulatory & $991,071(22.3)$ & $3,455,504(77.7)$ & $4,446,575$ \\
\hline Digestive & $609,727(24.4)$ & $1,888,117(75.6)$ & $2,497,844$ \\
\hline Endocrine System & $188,972(22.9)$ & $636,572(77.1)$ & 825,544 \\
\hline Eyes & $10,662(26.4)$ & $29,663(73.6)$ & 40,325 \\
\hline Female Reproductive System & $27,132(16.0)$ & $142,867(84.0)$ & 169,999 \\
\hline Hepatobiliary & $194,396(24.2)$ & $609,474(75.8)$ & 803,870 \\
\hline HIV & $17,490(22.3)$ & $61,097(77.7)$ & 78,587 \\
\hline Immunologic & $70,411(21.3)$ & $259,794(78.7)$ & 330,205 \\
\hline Infectious & $182,957(25.2)$ & $541,981(74.8)$ & 724,938 \\
\hline Injuries/Poisons & 116,601 (27.4) & $309,638(72.6)$ & 426,239 \\
\hline
\end{tabular}


Table 1 Demographics of patients admitted to the hospital for non-elective indications on a weekday compared with the weekends (Continued)

\begin{tabular}{lccc}
\hline Kidneys & $250,741(23.8)$ & $804,607(76.2)$ & $1,055,348$ \\
Male Reproductive System & $12,086(20.6)$ & $46,500(79.4)$ & 58,586 \\
Mental Health & $213,546(19.6)$ & $875,088(80.4)$ & $1,088,634$ \\
Musculoskeletal & $329,252(24.6)$ & $1,007,043(75.4)$ & $1,336,295$ \\
Myeloproliferative & $20,791(14.3)$ & $124,352(85.7)$ & 145,143 \\
Neonatal & $793,403(21.0)$ & $2,984,827(79.0)$ & $3,778,230$ \\
Other Health Factors & $33,873(15.5)$ & $184,566(84.5)$ & 218,439 \\
Otorhinolaryngology & $75,273(25.7)$ & $218,042(74.3)$ & 293,315 \\
Pregnancy And Childbirth & $478,320(21.6)$ & $1,736,923(78.4)$ & $2,215,243$ \\
Pre-Major Diagnostic Category & $3,621(27.2)$ & $9,677(72.8)$ & 13,298 \\
Respiratory & $731,704(25.2)$ & $2,175,813(74.8)$ & $2,907,517$ \\
Skin Or Breast & $145,317(23.6)$ & $470,145(76.4)$ & 615,462 \\
Trauma & $22,903(35.2)$ & $42,196(64.8)$ & 65,099 \\
\hline
\end{tabular}

Proportions reported in parenthesis unless otherwise noted.

Data are presented as number of discharges (percent) unless otherwise indicated. All comparisons by admission day are statistically significant, $\mathrm{p}<0.001$.

and error prone resulting in higher mortality following weekend admission.

\section{Methods}

\section{Data source}

We obtained all-payer discharge data from January 1, 2003, through December 31, 2008, via the Nationwide Inpatient Sample (NIS) of the Healthcare Cost and Utilization Project of the Agency for Healthcare Research and Quality. The NIS-the largest source of all-payer hospital discharge information in the United States-contains data from approximately 7 million to 8 million hospital stays per year in 1000 hospitals in over 30 states [4]. The hospitals sampled can vary from year to year but the sample approximates $20 \%$ of US community hospitals including large university hospitals and smaller regional facilities. The database provides information regarding patient demographics, socioeconomic factors, admission profiles, hospital profiles, state codes, discharge diagnoses, procedure codes, total charges, and vital status at hospital discharge. Along with other hospital discharge databases, the NIS has been used to review trends in surgical care and outcomes [5], volume outcome relationships [6], and disparities in care [7]. A data use agreement is held by the Agency for Healthcare Research and Quality, and our study was considered exempt by the Lahey Clinic Institutional Review Board.

The American Hospital Association (AHA) Annual Survey of Hospitals database was obtained in order to determine facility structural characteristics, service lines, staffing, and the presence of resident trainees at each hospital. The AHA database contains hospital-specific information on over 6000 hospitals and over 450 health- care systems, including 700 data elements [8]. The purpose of the AHA database is to generate a comprehensive and inclusive overview of hospitals while permitting the tracking of hospital performance over time. AHA data have been extensively used to study hospital-based outcomes [9], hospital policies [10], and reimbursement [11].

\section{Study population}

All patients discharged during the time frame sampled were included (both medical and surgical patients). We used the elective variable to exclude all patients with an admission for elective reasons and included only those patients with nonelective admission [4]. Thus, patients with emergency and urgent indications for admission were included in our study.

\section{Admission day}

The data set permits identification of admission day as a weekend or weekday. We recorded this variable as admitted during a weekend (i.e., Saturday or Sunday) or a weekday (i.e., Monday through Friday) [1,4].

\section{Covariates}

Our analysis adjusted for the following covariates: age, sex, race, income level, payer, major diagnostic categories (subgroupings of diagnosis-related groups), ${ }^{1}$ and the Charlson comorbidity index score. Age was included as a continuous variable. Sex was entered as a dichotomous variable. Race was divided into white, black, Hispanic, Asian or Pacific Islander, Native American, or other. Income level was categorized into quartiles per estimated median household income of residents in the patient's zip code [4]. The median income quartiles are classified 
Table 2 Hospital characteristics of patients admitted to the hospital for non-elective indications on a weekday compared with the weekends

\begin{tabular}{|c|c|c|c|}
\hline Hospital characteristic & Weekend & Weekday & All patients \\
\hline \multicolumn{4}{|l|}{ Hospital Control } \\
\hline Not-For-Profit, Nongovernment & $4,458,164(23.1)$ & $14,816,595(76.9)$ & $19,274,759$ \\
\hline Government & $837,958(23.0)$ & $2,811,631(77.0)$ & $3,649,589$ \\
\hline For-Profit, Investor Owned & $701,894(22.4)$ & $2,425,533(77.6)$ & $3,127,427$ \\
\hline \multicolumn{4}{|l|}{ Region } \\
\hline Northeast & $1,621,325(22.6)$ & $5,545,056(77.4)$ & $7,166,381$ \\
\hline Midwest & $954,215(23.2)$ & $3,156,368(76.8)$ & $4,110,583$ \\
\hline South & $1,791,365(22.8)$ & $6,079,594(77.2)$ & $7,870,959$ \\
\hline West & $1,631,111(23.6)$ & $5,272,741(76.4)$ & $6,903,852$ \\
\hline \multicolumn{4}{|l|}{ Urbanicity } \\
\hline Urban & $5,803,138(23.0)$ & $19,403,387(77.0)$ & $25,206,525$ \\
\hline Rural & $191,618(23.1)$ & $639,070(76.9)$ & 830,688 \\
\hline Missing & $3,260(22.4)$ & $11,302(77.6)$ & 14,562 \\
\hline \multicolumn{4}{|l|}{ Critical Access } \\
\hline Yes & $127,335(23.5)$ & $413,869(76.5)$ & 541,204 \\
\hline No & $5,829,471(23.0)$ & $19,507,489(77.0)$ & $25,336,960$ \\
\hline Missing & $41,210(23.7)$ & $132,401(76.3)$ & 173,611 \\
\hline \multicolumn{4}{|l|}{ Rural Referral Center } \\
\hline Yes & $402,736(23.1)$ & $1,341,355(76.9)$ & $1,744,091$ \\
\hline No & $5,569,464(23.0)$ & $18,631,686(77.0)$ & $24,201,150$ \\
\hline Missing & $25,816(24.2)$ & $80,718(75.8)$ & 106,534 \\
\hline \multicolumn{4}{|l|}{ Networked } \\
\hline Yes & $2,059,136(22.8)$ & $6,954,919(77.2)$ & $9,014,055$ \\
\hline No & $3,312,180(23.1)$ & $11,025,023(76.9)$ & $14,337,203$ \\
\hline Missing & $626,700(23.2)$ & $2,073,817(76.8)$ & $2,700,517$ \\
\hline \multicolumn{4}{|l|}{ Medical Surgical Intensive Care } \\
\hline Yes & $5,337,106(23.0)$ & $17,855,395(77.0)$ & $23,192,501$ \\
\hline No & $361,847(23.1)$ & $1,205,097(76.9)$ & $1,566,944$ \\
\hline Missing & $299,063(23.1)$ & $993,267(76.9)$ & $1,292,330$ \\
\hline \multicolumn{4}{|l|}{ Hospice Program } \\
\hline Yes & $1,801,819(23.1)$ & $5,984,198(76.9)$ & $7,786,017$ \\
\hline No & $3,831,348(22.9)$ & $12,878,741(77.1)$ & $16,710,089$ \\
\hline Missing & $364,849(23.5)$ & $1,190,820(76.5)$ & $1,555,669$ \\
\hline \multicolumn{4}{|l|}{ Cardiac Intensive Care } \\
\hline Yes & $3,866,696(23.0)$ & $12,975,259(77.0)$ & $16,841,955$ \\
\hline No & $1,792,240(23.2)$ & $5,946,583(76.8)$ & $7,738,823$ \\
\hline Missing & $339,080(23.1)$ & $1,131,917(76.9)$ & $1,470,997$ \\
\hline \multicolumn{4}{|l|}{ Pediatric Hospital } \\
\hline Yes & 4,187,982 (23.0) & $14,007,815(77.0)$ & $18,195,797$ \\
\hline No & $1,460,456(23.0)$ & $4,887,722(77.0)$ & $6,348,178$ \\
\hline Missing & $349,578(23.2)$ & $1,158,222(76.8)$ & $1,507,800$ \\
\hline
\end{tabular}




\begin{tabular}{|c|c|c|c|}
\hline \multicolumn{4}{|c|}{ Neonatal Intensive Care Unit } \\
\hline Yes & $3,023,448(23.0)$ & $10,147,156(77.0)$ & $13,170,604$ \\
\hline No & 2,619,397 (23.1) & $8,732,575(76.9)$ & $11,351,972$ \\
\hline Missing & $355,171(23.2)$ & $1,174,028(76.8)$ & $1,529,199$ \\
\hline \multicolumn{4}{|c|}{ Skilled Nursing Care } \\
\hline Yes & $1,540,309(23.1)$ & $5,141,015(76.9)$ & $6,681,324$ \\
\hline No & $4,087,411(23.0)$ & $13,707,285(77.0)$ & $17,794,696$ \\
\hline Missing & $370,296(23.5)$ & $1,205,459(76.5)$ & $1,575,755$ \\
\hline \multicolumn{4}{|c|}{ JCAHO Accreditation } \\
\hline Yes & $5,621,862(23.0)$ & $18,819,411(77.0)$ & $24,441,273$ \\
\hline No & $376,154(23.4)$ & $1,234,348(76.6)$ & $1,610,502$ \\
\hline Missing & 0 & 0 & 0 \\
\hline \multicolumn{4}{|c|}{ ACS Cancer Program } \\
\hline Yes & $3,542,482(23.0)$ & $11,860,791(77.0)$ & $15,403,273$ \\
\hline No & $2,455,534(23.1)$ & $8,192,968(76.9)$ & $10,648,502$ \\
\hline Missing & 0 & 0 & 0 \\
\hline \multicolumn{4}{|c|}{ Radiology, Photon Emission } \\
\hline Yes & $3,711,150(22.9)$ & $12,462,701(77.1)$ & $16,173,851$ \\
\hline No & $1,890,070(23.1)$ & $6,291,413(76.9)$ & $8,181,483$ \\
\hline Missing & $396,796(23.4)$ & $1,299,645(76.6)$ & $1,696,441$ \\
\hline \multicolumn{4}{|c|}{ Radiology, CT Scan } \\
\hline Yes & $4,065,915(23.0)$ & $13,630,554(77.0)$ & $17,696,469$ \\
\hline No & $1,546,023(23.1)$ & $5,133,106(76.9)$ & $6,679,129$ \\
\hline Missing & $386,078(23.0)$ & 1,290,099 (77.0) & $1,676,177$ \\
\hline \multicolumn{4}{|c|}{ Radiology, PET Scan } \\
\hline Yes & $2,200,507(22.8)$ & $7,437,175(77.2)$ & $9,637,682$ \\
\hline No & $3,416,652(23.1)$ & $11,381,603(76.9)$ & $14,798,255$ \\
\hline Missing & 380,857 (23.6) & $1,234,981(76.4)$ & $1,615,838$ \\
\hline \multicolumn{4}{|c|}{ Medical Beds } \\
\hline $0-20$ & 253,097 (23.1) & 844,804 (76.9) & $1,097,901$ \\
\hline $21-74$ & $779,721(23.0)$ & $2,603,130(77.0)$ & $3,382,851$ \\
\hline$\geq 74$ & $4,246,857(23.0)$ & $14,227,412(77.0)$ & $18,474,269$ \\
\hline Missing & $718,341(23.2)$ & 2,378,413 (76.8) & $3,096,754$ \\
\hline \multicolumn{4}{|c|}{ Total Hospital Beds } \\
\hline $0-53$ & $285,873(23.3)$ & $942,890(76.7)$ & $1,228,763$ \\
\hline $54-157$ & $935,537(23.1)$ & $3,109,974(76.9)$ & $4,045,511$ \\
\hline$\geq 157$ & $4,776,606(23.0)$ & $16,000,895(77.0)$ & $20,777,501$ \\
\hline Missing & 0 & 0 & 0 \\
\hline \multicolumn{4}{|c|}{ Pediatric Beds } \\
\hline 0 & $1,652,963(23.0)$ & $5,523,949(77.0)$ & $7,176,912$ \\
\hline $1-15$ & $1,766,364(23.1)$ & $5,872,580(76.9)$ & $7,638,944$ \\
\hline$\geq 16$ & $1,860,348(22.9)$ & 6,278,817 (77.1) & $8,139,165$ \\
\hline Missing & 718,341 (23.2) & 2,378,413 (76.8) & $3,096,754$ \\
\hline
\end{tabular}


Table 2 Hospital characteristics of patients admitted to the hospital for non-elective indications on a weekday compared with the weekends (Continued)

\begin{tabular}{lccc}
\hline Medical \& Dental Trainees & & & \\
0 & $2,911,505(23.2)$ & $9,646,519(76.8)$ & $12,558,024$ \\
$1-26$ & $1,250,566(23.1)$ & $4,158,208(76.9)$ & $5,408,774$ \\
$\geq 27$ & $1,835,945(22.7)$ & $6,249,032(77.3)$ & $8,084,977$ \\
Missing & 0 & 0 & $7,415,797$ \\
Physicians/Hospital Bed & & $5,705,164(76.9)$ & $7,341,414$ \\
$0-0.007$ & $1,710,633(23.1)$ & $5,654,953(77.0)$ & $10,972,232$ \\
$0.007-0.067$ & $1,686,461(23.0)$ & $8,445,660(77.0)$ & 322,332 \\
$\geq 0.067$ & $2,526,572(23.0)$ & $247,982(76.9)$ & $1,133,319$ \\
Missing & $74,350(23.1)$ & & $8,489,324$ \\
Nurses/Hospital Bed & & $873,025(77.0)$ & $16,106,800$ \\
$0-0.75$ & $260,294(23.0)$ & $6,544,395(77.1)$ & 322,332 \\
$0.75-1.359$ & $1,944,929(22.9)$ & $12,388,357(76.9)$ & $247,982(76.9)$ \\
$\geq 1.359$ & $3,718,443(23.1)$ & $74,350(23.1)$ & \\
Missing & & & \\
\hline
\end{tabular}

Proportions reported in parenthesis unless otherwise noted.

Data are presented as number of discharges (percent) unless otherwise indicated. All comparisons by admission day are statistically significant, $\mathrm{p}<0.001$, except for urbanicity ( $p$-value $=0.1195$ ).

as follows: $\$ 0$ to $\$ 38999, \$ 39000$ to $\$ 47999, \$ 48000$ to $\$ 62999$, and $\$ 63000$ or more [4].

Payer was recorded as follows: Medicare, Medicaid, private including health maintenance organization, selfpay, no charge, or other [4]. Major diagnostic categories were used to adjust for diagnoses and reflect larger groupings of diagnostic-related groups made available in the provided data set and downloadable for review from the US Department of Health and Human Services, Centers for Medicare and Medicaid Services [12]. Major diagnostic categories have been used to evaluate hospitalization risk [13], mortality risk [14], and other outcomes [15]. We also evaluated comorbidity with the Deyo modification of the Charlson comorbidity index [16]. Briefly, we ascertained the presence of 17 comorbid conditions and then weighted them according to the

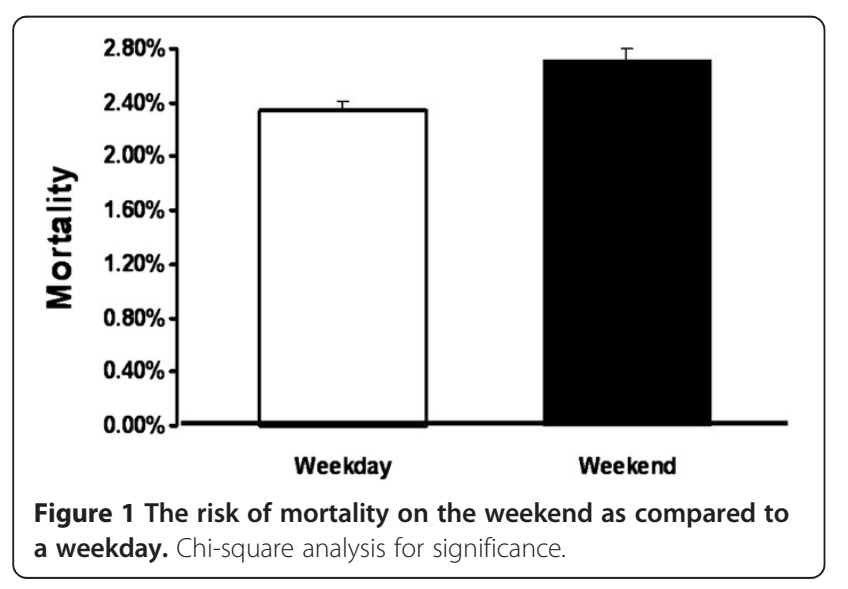

original report. An elevated Charlson comorbidity index score has been demonstrated to correlate with higher mortality rate [17].

Hospital bed size categories were obtained from the American Hospital Association Annual Survey of Hospitals and based on the number of short-term acute care beds.

\section{Staffing}

Staffing levels were obtained from the American Hospital Association Annual Survey of Hospitals [8]. We analyzed the role of full-time registered nurses and full-time physicians on mortality by developing ratios of either nurse or physician per hospital bed. We categorized these two variables into tertiles, low, medium, or high.

\section{Presence of resident trainees}

The teaching status of the hospital was obtained from the American Hospital Association Annual Survey of Hospitals [8]. Presence of resident trainees was categorized into tertiles. Given that half of all facilities had no residents, this was the lowest tertile. The middle tertile included 1-26 resident trainees. The highest tertile included greater than or equal to 27 resident trainees.

\section{Outcome}

The data set permits identification of vital status at the time of discharge. The variable is coded as died during hospitalization or did not die during hospitalization. Deaths that occurred after discharge are not identifiable from our data set [4]. 
Table 3 Multivariate analysis of mortality for non-elective admissions

Characteristic
Age
Male
Female
Race
White
Asian
Black
Hispanic
Native American
Other

Median Household Income

0-25th Percentile

26-50th Percentile

51-75th Percentile

76-100th Percentile

Primary Payer

Private

Medicaid

Medicare

None

Other

Self-Pay

Comorbidity Score

Admission Day

Weekday

Weekend

MDC

Nervous System

Alcohol/Drug Use

Burns

Circulatory

Digestive

Endocrine System

Eyes

Female Reproductive System

Hepatobiliary

HIV

Immunologic

Infectious

Injuries/Poisons

Kidneys

Male Reproductive System

Mental Health
Odds ratio

1.04

Reference

0.9

$0.8-0.9$

0.0001

Reference

\begin{tabular}{|c|c|c|}
\hline 1.0 & $1.0-1.0$ & 0.6 \\
\hline 1.0 & $1.0-1.0$ & 0.3 \\
\hline 0.9 & $0.9-0.9$ & 0.0001 \\
\hline 1.0 & $0.9-1.0$ & 0.4 \\
\hline 1.1 & $1.1-1.1$ & 0.0001 \\
\hline
\end{tabular}

Reference

0.98

0.97-0.99

0.0001

0.96

0.96-0.97

0.0001

0.95

0.94-0.96

0.0001

Reference

$\begin{array}{ccc}1.2 & 1.2-1.3 & 0.0001 \\ 1.0 & 1.0-1.0 & 0.1 \\ 0.9 & 0.8-0.9 & 0.0001 \\ 1.4 & 1.4-1.5 & 0.0001 \\ 1.5 & 1.5-1.5 & 0.0001 \\ 1.2 & 1.2-1.2 & 0.0001\end{array}$

Reference

Reference

0.1

1.8

0.5

0.5

0.4

0.1

0.4

0.7

1.1

0.4

3.3

0.5

0.6

0.3

0.1
0.1-0.1

0.0001

1.6-1.9

0.0001

0.5-0.5

0.5-0.5

0.4-0.4

0.1-0.1

0.4-0.5

0.7-0.7

1.1-1.1

0.4-0.4

3.2-3.3

0.5-0.5

0.5-0.6

0.3-0.3

0.1-0.1
0.0001

0.0001

0.0001

0.0001

0.0001

0.0001

0.0001

0.0001

0.0001

0.0001

0.0001

0.0001

0.0001 
Table 3 Multivariate analysis of mortality for non-elective admissions (Continued)

\begin{tabular}{|c|c|c|c|}
\hline Musculoskeletal & 0.3 & $0.3-0.3$ & 0.0001 \\
\hline Myeloproliferative & 1.5 & $1.5-1.6$ & 0.0001 \\
\hline Neonatal & 1.4 & $1.4-1.4$ & 0.0001 \\
\hline Other Health Factors & 0.3 & $0.3-0.3$ & 0.0001 \\
\hline Otorhinolaryngology & 0.2 & $0.2-0.2$ & 0.0001 \\
\hline Pregnancy And Childbirth & 0.0 & $0.0-0.0$ & 0.0001 \\
\hline Pre-Major Diagnostic Category & 1.4 & $1.3-1.5$ & 0.0001 \\
\hline Respiratory & 1.2 & $1.2-1.2$ & 0.0001 \\
\hline Skin Or Breast & 0.2 & $0.2-0.2$ & 0.0001 \\
\hline Trauma & 5.1 & $5.0-5.3$ & 0.0001 \\
\hline \multicolumn{4}{|l|}{ Hospital Control } \\
\hline Not-For-Profit, Nongovernment & Reference & & \\
\hline Government & 1.1 & $1.1-1.1$ & 0.0001 \\
\hline For-Profit, Investor Owned & 0.97 & $0.96-0.99$ & 0.0001 \\
\hline \multicolumn{4}{|l|}{ Region } \\
\hline Northeast & Reference & & \\
\hline Midwest & 0.9 & $0.9-0.9$ & 0.0001 \\
\hline South & 0.9 & $0.9-0.9$ & 0.0001 \\
\hline West & 0.95 & $0.94-0.96$ & 0.0001 \\
\hline Urbanicity & 1.01 & $1.0-1.02$ & 0.03 \\
\hline \multicolumn{4}{|l|}{ Critical Access } \\
\hline Yes & 0.8 & $0.8-0.8$ & 0.0001 \\
\hline Missing & 0.8 & $0.8-0.9$ & 0.0001 \\
\hline \multicolumn{4}{|l|}{ Rural Referral Center } \\
\hline Yes & 1.1 & $0.1-1.1$ & 0.0001 \\
\hline Missing & 1.4 & $1.4-1.6$ & 0.0001 \\
\hline \multicolumn{4}{|l|}{ Networked } \\
\hline Yes & 0.99 & $0.99-1.00$ & 0.02 \\
\hline Missing & 0.9 & $0.9-0.9$ & 0.0001 \\
\hline Medical Surgical Intensive Care & 1.0 & $1.0-1.0$ & 0.5 \\
\hline Hospice Program & 1.0 & $1.0-1.0$ & 0.5 \\
\hline Cardiac Intensive Care & 1.04 & $1.03-1.05$ & 0.0001 \\
\hline Pediatric Hospital & 0.97 & $0.96-0.99$ & 0.0001 \\
\hline Pediatric Intensive Care & 1.05 & $1.04-1.06$ & 0.0001 \\
\hline Neonatal Intensive Care Unit & 1.0 & $1.0-1.0$ & 0.4 \\
\hline Skilled Nursing Care & 1.04 & $1.03-1.05$ & 0.0001 \\
\hline JCAHO Accreditation & 1.04 & $1.02-1.05$ & 0.0001 \\
\hline ACS Cancer Program & 1.0 & $1.0-1.0$ & 0.1 \\
\hline Radiology, Photon Emission & 0.98 & $0.97-0.99$ & 0.0001 \\
\hline Radiology, CT Scan & 0.9 & $0.9-0.9$ & 0.0001 \\
\hline Radiology, PET Scan & 1.1 & $1.1-1.1$ & 0.0001 \\
\hline \multicolumn{4}{|l|}{ Medical Beds } \\
\hline $0-20$ & Reference & & \\
\hline $21-74$ & 0.8 & $0.8-0.8$ & 0.0001 \\
\hline
\end{tabular}


Table 3 Multivariate analysis of mortality for non-elective admissions (Continued)

\begin{tabular}{|c|c|c|c|}
\hline$\geq 74$ & 0.8 & $0.8-0.8$ & 0.0001 \\
\hline Missing & 0.8 & $0.8-0.8$ & 0.0001 \\
\hline \multicolumn{4}{|c|}{ Total Hospital Beds } \\
\hline $0-53$ & Reference & & \\
\hline $54-157$ & 1.0 & $1.0-1.0$ & 0.3 \\
\hline$\geq 157$ & 1.1 & $1.0-1.1$ & 0.0001 \\
\hline \multicolumn{4}{|l|}{ Pediatric Beds } \\
\hline 0 & Reference & & \\
\hline $1-15$ & 1.0 & $1.0-1.0$ & 0.1 \\
\hline$\geq 16$ & 0.1 & $1.0-1.1$ & 0.0001 \\
\hline \multicolumn{4}{|c|}{ Medical \& Dental Trainees } \\
\hline 0 & Reference & & \\
\hline $1-26$ & 1.03 & $1.02-1.04$ & 0.0001 \\
\hline$\geq 27$ & 1.05 & $1.04-1.07$ & 0.0001 \\
\hline \multicolumn{4}{|c|}{ Physicians/Hospital Bed } \\
\hline $0-0.007$ & Reference & & \\
\hline $0.007-0.067$ & 0.9 & $0.9-0.9$ & 0.0001 \\
\hline$\geq 0.067$ & 0.9 & $0.9-0.9$ & 0.0001 \\
\hline \multicolumn{4}{|c|}{ Nurses/Hospital Bed } \\
\hline $0-0.75$ & Reference & & \\
\hline $0.75-1.359$ & 0.9 & $0.9-1.0$ & 0.0001 \\
\hline$\geq 1.359$ & 0.9 & $0.9-0.9$ & 0.0001 \\
\hline
\end{tabular}

\section{Statistical analysis}

Statistical analyses were performed using SAS statistical software, version 9.2 (SAS Institute Inc, Cary, North Carolina). We analyzed univariate associations with patient admission day (weekend vs. weekday) using $t$ tests for continuous variables and $\chi 2$ tests for categorical variables. Results were considered statistically significant at $\mathrm{p}<0.05$, and all statistical tests were 2-tailed. We included all covariates in our regression model. The analyses were conducted with and without missing data. To confirm results, we performed imputation of missing data using the multiple imputation procedure from SAS Institute Inc [18]. Imputation substitutes missing values with plausible values that characterize the uncertainty regarding the missing data $[19,20]$. This process results in valid statistical inferences that properly reflect the uncertainty due to missing values, for example, confidence

Table 4 Adjusted odds of mortality with test for interaction between full-time resident trainees and day of admission

\begin{tabular}{lccc}
\hline & \multicolumn{3}{c}{ Full time residents } \\
\cline { 2 - 4 } Day of Admission & $\mathbf{0}$ (Referent) & $\mathbf{1 - 2 6}$ & $\mathbf{2} \mathbf{2 7}$ \\
\hline Weekday & 1.0 & 1.03 & 1.05 \\
Weekend & 1.0 & 1.11 & 1.17 \\
\hline
\end{tabular}

intervals with the correct probability coverage. The multiply imputed dataset was then analyzed by using standard logistic regression for the complete data.

We tested for interactions between staffing levels, resident trainees and admission day on mortality in the regression analysis.

\section{Results}

Cohort

Data were available for 48,253,968 patient discharges during the six-year study period, of which 26,038,921 were non-elective. Demographics, patient characteristics, and comorbidity are listed in Table 1 in relation to day of admission. In addition, Table 2 lists the hospital characteristics, staffing levels, and other AHA variables included in our analysis.

\section{Univariate analysis}

The relative risk of mortality was $15 \%$ higher on the weekend as compared to a weekday (Figure 1). Patients admitted on the weekend were on average older (47.9 vs. 46.9 years) than those admitted during a weekday. Males were more likely to be admitted on a weekend than females (23.3\% vs. $22.8 \%)$. Whites were less likely to be admitted on a weekend than all other racial groups. Lower income categories were more likely to be admitted on a 
weekend than the highest quartile group. Self-paying and patients without health insurance were more likely to be admitted on the weekend $(25.4 \%$ and $25.0 \%$, respectively) than patients with other primary methods of payment. On average, patients admitted during the weekend had a higher comorbidity score (Tables 1 and 2).

\section{Multivariate analysis}

After adjusting for diagnosis, age, sex, race, income level, payer, comorbidity, and weekend admission the overall odds of mortality was higher for patients in hospitals with fewer nurses and staff physicians. Conversely, hospitals with more physicians or nurses per hospital bed were associated with a $10 \%$ reduction in mortality after a weekend admission (Odds Ratio $=0.9$ : CI 0.9-0.9). In addition, mortality was higher for patients in hospitals with more beds (Odds Ratio $=1.1$ : CI 1.0-1.1) and more resident trainees (Odds Ratio $=1.05:$ CI 1.04-1.07) $($ Table 3) .

\section{Tests for interactions}

We identified no interactions between staffing (registered nurse or physician) and admission day on mortality. However, we identified significant interactions for the presence of resident trainees. Mortality following a weekend admission to a hospital with the highest tertile of resident trainees was $17 \%$ higher (odds ratios 1.17 vs. 1.05) than hospitals with no resident trainees $(\mathrm{p}<0.001)$ (Table 4) after adjusting for all covariates outlined in Table 3. This excess mortality following a weekend admission is significantly higher than the $5 \%$ increase observed between tertiles when admission occurs on a weekday (p-value for interaction $<0.001$ ).

\section{Discussion}

Using national all-payer discharge data, we confirmed significant differences in inpatient mortality as a function of day of admission. We also identified associations between staffing levels (both nursing and physician) and the outcome of mortality. However, the presence of resident physicians did not mitigate the effect of weekend day admission on mortality. Instead we found the reverse, a direct correlation between the presence of resident trainees and higher mortality. Most importantly, we identified interactions between admission day and the presence of resident trainees on the likelihood of inpatient mortality. These results may indicate that inadequate resident supervision by staff physicians during weekend patient care may adversely impact patient outcomes.

A prior study [21] did identify increased mortality at hospitals classified as "teaching" in the one state and with a smaller sample of medical diagnoses. However given the limited number of diagnoses and sample size in that study [21], we performed the study with a larger group of diagnoses, with nonelective admissions, and with a national sample of patients. In emergency settings, we hypothesized that trainees provide continuous on-site patient care, which is both invaluable and timely. Hospitals with residency training programs would thus harness the benefits of a team structure to care, endorsing multiple patient evaluations and re-evaluations and a care system focused on checks and balances. For example, in the setting of an intensive care unit, Poses and colleagues reported that the combined judgment of two junior or senior house officers was as good as that of the attending physician in managing the intensive care unit patient [22]. In a review by Kupersmith, teaching hospitals demonstrated better-quality measures, particularly measures of process, than did nonteaching hospitals [23]. The attention to detail inherent in a setting where trainees are present, general use of current medical literature to guide clinical decision making, and more frequent and thorough case reviews should contribute to a lower incidence of adverse occurrences [24]. In fact, improved outcomes have been demonstrated in some studies evaluating the role of residency programs. In a review of outcomes based on teaching status, lower riskadjusted mortality was noted in major teaching hospitals for elderly patients with common conditions such as acute myocardial infarction, congestive heart failure, and pneumonia [25]. Contrary to these prior findings, our study of nonelective admissions to acute care hospitals revealed the opposite effect. It should be understood however, that our study was specific to nonelective admissions on the weekend alone.

In addition to the role of trainees on admission day outcomes, the difference in mortality following weekend admission as compared to weekdays was noted at hospitals without resident trainees as well. However, the mortality differences based on day of admission were particularly augmented at those hospitals in which residents train. We used tertiles for cutoffs of trainees to understand this effect because of the lack of data demonstrating any particular trainee presence as correlated with outcome. We noted that although mortality was higher on weekends whether or not a facility had a training program, that the mortality difference was substantially higher if the patient was admitted on a weekend to a facility with more resident trainees. The consistent elevation in mortality based on a weekend admission however implicate a more central structural or process measure, which is more fundamental than the role of trainees. It is likely that a common factor that is linked to residency training is critical to this admission day outcomes difference. This common factor may be related to supervision, provider staffing, care integration, or other specific provider factors. Our data did demonstrate higher mortality after weekend admission for patients in hospitals with fewer nurses and staff physicians, 
but this staffing finding is contrary to the resident trainee effect. Thus, although there is a value to more staff nurses [26], and physicians, more resident trainees seem to have the opposite effect on mortality. This conflicting finding leads us to more questions than answers regarding the role of staffing on healthcare outcomes.

Our study has limitations based on the data used for analysis. Although the administrative data used in our study are population based, there is the potential for information and misclassification bias. It is unlikely that mortality or day of admission were improperly abstracted from the medical record; however, staffing levels and the presence of trainees may not be as rigorously documented. The data are limited to inpatient mortality and there is not assumption that the mortality trend persists beyond the inpatient setting. It should be understood that our data do not tie a direct link between the care provided by a trainee and the outcome of mortality. Our data only demonstrate that the increased mortality noted when patients are admitted on a weekend is worse when a facility has resident trainees. In addition to this concern, it is possible that patients admitted during the weekend have more comorbidities or potentially more severe illnesses at hospitals with resident trainees. Although we have adjusted for comorbidity, an assessment of disease severity at presentation is not possible with the available data. However, others have not identified significant differences in healthcare resource groupings indicating high comorbidity or complications at National Health Service hospitals in England across admission day [2]. Thus, despite these limitations, the strength of our study is in the large population studied implying that the results are representative and generalizable to the nation.

\section{Conclusions}

In conclusion, our data indicate that the role of staffing and resident trainees is an important area of focus for patient safety. Our study does not argue against a positive benefit of resident trainees to the sponsoring institution, the local community, affiliated academic health center, and the greater community [24]. Rather, our data suggest that the role of resident supervision may be an important target for quality weekend medical care. Although a causal link between supervision and outcome can not be identified in this study, our data do demonstrate that hospitals with many trainees have the highest mortality burden following a weekend admission. An assessment of rounding practices, trainee-directed procedures, and/or call routines of supervising physicians would help answer questions raised by our study. Following these assessments, strategies can be developed and implemented to standardize care across admission day.

\section{Competing interests}

Dr. Schoetz is the Chair of Medical Education at Lahey Hospital and Medical Center.

\section{Authors' contributions}

Conception and Design: RR, JN, Acquisition of Data: RR, Analysis and Interpretation of Data: RR, JN, DJS, TER, PLR, PWM, Drafting the Article: RR, JN, Critical Revision: RR, JN, DJS, TER, PLR, PWM, Final Manuscript Approval: RR, $J N$, DJS, TER, PLR, PWM. All authors read and approved the final manuscript.

\section{Acknowledgements \\ Financial support}

Wise Educational Grant.

Received: 14 August 2013 Accepted: 27 December 2013

Published: 8 January 2014

\section{References}

1. Ricciardi R, Roberts PL, Read TE, Baxter NN, Marcello PW, Schoetz DJ: Mortality rate after nonelective hospital admission. Arch Surg 2011, 146:545-551.

2. Mohammed MA, Sidhu KS, Rudge G, Stevens AJ: Weekend admission to hospital has a higher risk of death in the elective setting than in the emergency setting: a retrospective database study of national health service hospitals in England. BMC Health Serv Res 2012, 12:87.

3. Wong HJ, Morra D: Excellent hospital care for all: open and operating 24/7. J Gen Intern Med 2011, 26:1050-1052.

4. Healthcare Cost and Utilization Project (HCUP): Overview of the nationwide inpatient sample; July 2010. Agency for healthcare research and quality Web site. www.hcup-us.ahrq.gov/nisoverview.jsp. Accessed November 18, 2013.

5. Dimick JB, Wainess RM, Cowan JA, Upchurch GR Jr, Knol JA, Colletti LM: National trends in the use and outcomes of hepatic resection. J Am Coll Surg 2004, 199:31-38.

6. Ricciardi R, Virnig BA, Ogilvie JW Jr, Dahlberg PS, Selker HP, Baxter NN: Volume-outcome relationship for coronary artery bypass grafting in an era of decreasing volume. Arch Surg 2008, 143:338-344.

7. Ricciardi R, Selker HP, Baxter NN, Marcello PW, Roberts PL, Virnig BA: Disparate use of minimally invasive surgery in benign surgical conditions. Surg Endosc 2008, 22:1977-1986.

8. American Hospital Association: Hospital connect online store. Accessed November 8, 2005. 2005. Health Forum. http://ams.aha.org/eweb/StartPage. aspx. Accessed February 16, 2014

9. Elting LS, Pettaway C, Bekele BN, Grossman HB, Cooksley C, Avritscher EB, Saldin K, Dinney CP: Correlation between annual volume of cystectomy, professional staffing, and outcomes: a statewide, population-based study. Cancer 2005, 104:975-984.

10. Powner DJ, Hernandez M, Rives TE: Variability among hospital policies for determining brain death in adults. Crit Care Med 2004, 32:1284-1288.

11. Irvin CB, Fox JM, Pothoven K: Financial impact on emergency physicians for nonreimbursed care for the uninsur. Ann Emerg Med 2003, 42:571-576.

12. US Department of Health and Human Services: Centers for Medicare and Medicaid services, details for fiscal year 2008 major diagnostic categories file, Centers for Medicare and Medicaid services. http://www.cms.gov/Medicare/ Medicare-Fee-for-Service-Payment/AcutelnpatientPPS/Acute-Inpatient-Filesfor-Download-Items/CMS1247844.html. Accessed November 18, 2013.

13. Muennig $\mathrm{P}$, Jia $\mathrm{H}$, Khan $\mathrm{K}$, Pallin DJ: Ascertaining variation in hospitalization risk among immigrants using small area analysis. Prev Med 2006, 43:145-149.

14. Durairaj L, Will JG, Torner JC, Doebbeling BN: Prognostic factors for mortality following interhospital transfers to the medical intensive care unit of a tertiary referral center. Crit Care Med 2003, 31:1981-1986.

15. Brameld K, Holman D, Moorin R: Possession of health insurance in Australia-how does it affect hospital use and outcomes? J Health Serv Res Policy 2006, 11:94-100.

16. Deyo RA, Cherkin DC, Ciol MA: Adapting a clinical comorbidity index for use with ICD-9-CM administrative databases. J Clin Epidemiol 1992, 45:613-619.

17. Pompei $P$, Charlson ME, Ales K, MacKenzie CR, Norton M: Relating patient characteristics at the time of admission to outcomes of hospitalization. J Clin Epidemiol 1991, 44:1063-1069. 
18. SAS Institute Inc: The missing imputation procedure. SAS Institute Inc Web site. http://support.sas.com/documentation/. Accessed February 16, 2014

19. Schafer JL: Multiple imputation: a primer. Stat Methods Med Res 1999 , 8:3-15. Review.

20. Rubin DB, Schenker N: Multiple imputation in health-care databases: an overview and some applications. Stat Med 1991, 10:585-598

21. Cram P, Hillis SL, Barnett M, Rosenthal GE: Effects of weekend admission and hospital teaching status on in-hospital mortality. Am J Med 2004, 117:151-157.

22. Poses RM, Bekes C, Winkler RL, Scott WE, Copare FJ: Are two (inexperienced) heads better than one (experienced) head? Averaging house officers' prognostic judgments for critically ill patients. Arch Intern Med 1990, 150:1874-1878.

23. Kupersmith J: Quality of care in teaching hospitals: a literature review. Acad Med 2005, 80:458-466.

24. Pugno PA, Gillanders WR, Kozakowski SM: The direct, indirect, and intangible benefits of graduate medical education programs to their sponsoring institutions and communities. J Grad Med Educ 2010, 2:154-159.

25. Ayanian JZ, Weissman JS: Teaching hospitals and quality of care: a review of the literature. Milbank Q 2002, 80:569-593. v. Review.

26. Aiken LH, Clarke SP, Sloane DM, Sochalski J, Silber JH: Hospital nurse staffing and patient mortality, nurse burnout, and job dissatisfaction. JAMA 2002, 288:1987-1993.

doi:10.1186/1472-6920-14-4

Cite this article as: Ricciardi et al.: Is the presence of medical trainees associated with increased mortality with weekend admission? BMC Medical Education 2014 14:4.

\section{Submit your next manuscript to BioMed Central and take full advantage of:}

- Convenient online submission

- Thorough peer review

- No space constraints or color figure charges

- Immediate publication on acceptance

- Inclusion in PubMed, CAS, Scopus and Google Scholar

- Research which is freely available for redistribution 